PROCEEDINGS OF THE

AMERICAN MATHEMATICAL SOCIETY

Volume 129, Number 5, Pages 1391-1393

S 0002-9939(00)05711-7

Article electronically published on October 20, 2000

\title{
NON-HYPERBOLIC COMPLEX SPACE WITH A HYPERBOLIC NORMALIZATION
}

\author{
SHULIM KALIMAN AND MIKHAIL ZAIDENBERG
}

(Communicated by Steven R. Bell)

\begin{abstract}
We construct an example of a non-hyperbolic singular projective surface $X$ whose normalization $V$ is the square of a genus 3 curve $C$ and hence, hyperbolic.
\end{abstract}

Let $C$ be a smooth irreducible projective curve of genus $g \geq 2$. Then the smooth projective surface $V=C \times C$ is Kobayashi hyperbolic, that is, the Kobayashi pseudodistance on it is a distance [2]. Let $V \hookrightarrow \mathbb{P}^{N}$ be a projective embedding. Consider a generic projection $\pi: V \rightarrow \mathbb{P}^{3}$. By Bertini's theorem, the singular locus $S$ (i.e. the closure of the set of double points) of the image surface $X=\pi(V) \subset \mathbb{P}^{3}$ is an irreducible curve, and $\pi: V \rightarrow X$ is a normalization map (see [4]). The question arises whether the surface $X$ is also hyperbolic. The answer is positive [5], and hence by the stability of hyperbolicity theorem [6], any (smooth) surface $X^{\prime}$ in $\mathbb{P}^{3}$ close enough to $X$ is hyperbolic, as well. In that way examples of degree 32 smooth hyperbolic surfaces in $\mathbb{P}^{3}$ were produced [5].

By Proposition 1.1 in [5], hyperbolicity of a (singular) surface $X$ as above is equivalent to hyperbolicity of its double curve $S$. Actually, in [5] it is shown that the geometric genus of the curve $S$ is $\geq 225$, which provides that $X$ is hyperbolic.

On the other hand, by the Kobayashi-Kwack theorem [2, 3], a normalization of a hyperbolic complex space is also hyperbolic. In this note we give an example which shows that in general, the converse is not true. To describe this example, denote by $C$ the Fermat quartic $x^{4}+y^{4}+z^{4}=0$ in $\mathbb{P}^{2}$. Then the Cartesian square $V=C \times C \subset \mathbb{P}^{2} \times \mathbb{P}^{2} \hookrightarrow \mathbb{P}^{8}$ (the Segre embedding) is a smooth surface of degree 32 in $\mathbb{P}^{8}$. We construct a singular projective surface $X$ whose normalization is $V$, which has a fibration $X \rightarrow C$ over $C$ with general fibre isomorphic to $C$ and with four degenerate fibres $C_{i}, i=1, \ldots, 4$, isomorphic to $\mathbb{P}^{1}$. The "double curve" $S=C_{1} \cup \cdots \cup C_{4} \subset X$ of $X$ is neither irreducible nor hyperbolic, in contrast with the situation studied in [5]. Thus, the assumption in [5] that the projection $\pi$ is generic, is likely to be essential to provide hyperbolicity of the image surface $X=\pi(V)$.

Actually, in our example the surface $X$ does not appear as a projection of $V=$ $C \times C$; but it has a natural embedding into a four-dimensional Brauer-Severi variety $Y$ (see [1]) which is a smooth projective fiber bundle over $\mathbb{P}^{2}$ with general fibre $\mathbb{P}^{2}$.

Received by the editors July 30, 1999.

2000 Mathematics Subject Classification. Primary 32H15, 32H20.

(C)2000 American Mathematical Society 
More precisely, let the surface $V=C \times C \subset \mathbb{P}^{2} \times \mathbb{P}^{2}$ be given as

$$
V=\left\{\begin{aligned}
y^{4}+x^{4}+z^{4} & =0 \\
u^{4}+v^{4}+w^{4} & =0 .
\end{aligned}\right.
$$

Evidently, it is hyperbolic; indeed, $C$ is a smooth genus 3 curve.

Set $C_{1}=C \backslash\{z=0\}$ and $C_{2}=C \backslash\{x=0\}$. Let $q: E \rightarrow C$ be an algebraic fiber bundle over $C$ with the fiber $\mathbf{P}^{2}$ such that $q^{-1}\left(C_{k}\right) \cong C_{k} \times F_{k}$ where $F_{k} \cong \mathbf{P}^{2}$ with a homogeneous coordinate system $\left(u_{k}: v_{k}: w_{k}\right), k=1,2$, and the transition mapping is given in $q^{-1}\left(C_{1} \cap C_{2}\right)$ as follows: $\left(u_{2}: v_{2}: w_{2}\right)=\left(z u_{1}: x v_{1}: x w_{1}\right)$. Let $X \subset E$ be the surface defined by the equations

$$
X_{1}=X \cap q^{-1}\left(C_{1}\right)=\left\{\begin{aligned}
y^{4}+x^{4}+z^{4} & =0, \\
z^{4} u_{1}^{4}+y^{4}\left(v_{1}^{4}+w_{1}^{4}\right) & =0
\end{aligned}\right.
$$

and

$$
X_{2}=X \cap q^{-1}\left(C_{2}\right)=\left\{\begin{aligned}
y^{4}+x^{4}+z^{4} & =0, \\
x^{4} u_{2}^{4}+y^{4}\left(v_{2}^{4}+w_{2}^{4}\right) & =0 .
\end{aligned}\right.
$$

Then the intersection $S:=X \cap\{y=0\}$ consists of four disjoint smooth rational curves (whereas any other fibre of the natural projection $X \rightarrow C$, that is, the restriction to $X$ of the projection of the Cartesian square $\mathbb{P}^{2} \times \mathbb{P}^{2}$ to the first factor, is isomorphic to the curve $C$ ). Thus, the surface $X$ is not hyperbolic (cf. Remark 1 below).

Put $V_{1}=V \backslash\{z=0\}, V_{2}=V \backslash\{x=0\}$, and consider further the morphisms $\nu_{i}: V_{i} \rightarrow X_{i}, i=1,2$, given as

$$
\left(u_{1}: v_{1}: w_{1}\right)=(y u: z v: z w) \quad \text { resp., }\left(u_{2}: v_{2}: w_{2}\right)=(y u: x v: x w) .
$$

It is easily seen that these formulas define a birational morphism $\nu: V \rightarrow X$ which makes $V$ a normalization of $X$. Indeed, since $V$ is a smooth surface, $\nu$ can be factorized through the normalization $\nu^{\prime}: V^{\prime} \rightarrow X$ of $X$, that is, $\nu=\mu \circ \nu^{\prime}$ where $\mu: V \rightarrow V^{\prime}$ is a birational morphism. It is easily seen that $\mu$ is a bijection, and then by Zariski's Main Theorem, it is an isomorphism. Thus, $\nu: V \rightarrow X$ is a normalization of $X$. This gives a desired example.

Remarks. 1. In fact, the surface $X$ is hyperbolic modulo the "double curve" $S$. This follows from the fact that any holomorphic disc $f: \Delta \rightarrow X$ (where $\Delta$ denotes the unit disc) whose image is not contained in $S$ can be lifted to the normalization, that is, there exists a holomorphic $\operatorname{disc} \widehat{f}: \Delta \rightarrow V$ such that $f=\nu \circ \widehat{f}$. Hence, since $V$ is hyperbolic, the Kobayashi-Royden pseudometric on $X$ can be estimated from below outside of the directions tangent to $S$.

2. We can easily get a similar example of a non-hyperbolic affine algebraic surface which has a smooth hyperbolic affine normalization. Indeed, let $V_{0} \subset \mathbb{C}^{4}$ resp., $X_{0} \subset \mathbb{C}^{4}$ be the surface given by the equations

$$
\left\{\begin{array}{l}
y^{4}+x^{4}+1=0, \\
u^{4}+v^{4}+1=0
\end{array}\right.
$$

resp.,

$$
\left\{\begin{aligned}
y^{4}+x^{4}+1 & =0 \\
u^{4}+y^{4}\left(v^{4}+1\right) & =0
\end{aligned}\right.
$$


Then, as above, the restriction to $V_{0}$ of the birational morphism

$$
\sigma: \mathbb{C}^{4} \rightarrow \mathbb{C}^{4}, \sigma:(x, y, u, v) \longmapsto(x, y, y u, v)
$$

(which consists of blowing up with center at the plane $y=u=0$ and then deleting the proper transform of the divisor $y=0$ ) makes $V_{0}$ a normalization of $X_{0}$. The intersection $S_{0}:=X_{0} \cap\{y=0\}$ consists of four complex affine lines $\simeq \mathbb{C}$ and hence, the surface $X_{0}$ is not hyperbolic, whereas its normalization $V_{0}$ is hyperbolic being the Cartesian square of a hyperbolic affine curve $C_{0}=\left\{x^{4}+y^{4}+1=0\right\}$.

\section{REFERENCES}

[1] A. Grothendieck, Le groupe de Brauer. Dix exposés sur la cohomologie des schémas, 46-65 Mason and Cie, Paris, North-Holland Publ. Co., Amsterdam, 1968. MR 39:5586a

[2] Sh. Kobayashi, Hyperbolic manifolds and holomorphic mappings. Marcel Dekker, Inc., New York, 1970. MR 43:3503

[3] M.H. Kwack, Generalization of the big Picard theorem. Ann. Math. 90 (1969), 9-22. MR 39:4445

[4] B. Moishezon, Complex surfaces and connected sums of complex projective planes. Lect. Notes in Math. 603. Springer, Berlin, 1977. MR 58:10931

[5] B. Shiffman and M. Zaidenberg, Two classes of hyperbolic surfaces in $\mathbb{P}^{3}$. International J. Math. 11:1, 2000 (to appear); preprint MPI-1998-129, 33p., http://www.mpim-bonn.mpg.de/.

[6] M. Zaidenberg, Stability of hyperbolic embeddedness and construction of examples. Math. USSR Sbornik 135 (1988), 361-372, 415. MR 89f:32047

Department of Mathematics and Computer Science, University of Miami, Coral Gables, Florida 33124

E-mail address: kaliman@cs.miami.edu

Université Grenoble I, Institut Fourier, UMR 5582 CNRS-UJF, BP 74, 38402 St. Martin d'Hères CÉdex, France

E-mail address: zaidenbe@ujf-grenoble.fr 